\title{
Wegener Granulomatosis: Case Report and Brief Literature Review
}

\author{
Khaled Shafiei, MD, Euil Luther, MD, Michael Archie, MD, Janis Gulick, MD, and \\ Marjorie R. Fowler, MD
}

Wegener granulomatosis (WG) is an uncommon condition characterized by necrotizing granulomatosis of the upper and lower respiratory tract and glomerulonephritis. We describe a case of a $42-$ year-old white man with symptoms of pansinusitis who gradually developed more symptoms of WG. This case emphasizes early diagnosis and treatment to avoid further complications.

\section{Case Report}

The patient was a 42-year-old white man with a history of sinusitis for almost 2 years. He was treated with different antibiotics as an outpatient without any improvement. He developed a lung mass on chest radiograph, so he was referred to the surgical service for biopsy. On the day of the planned biopsy, the patient was febrile with an elevated white blood cell count (WBC). He also had severe headache; therefore, biopsy was canceled and the patient was transferred to the Family Practice team.

At admission, his temperature was $98.8^{\circ} \mathrm{F}$, pulse was 90 beats/min, respiratory rate was 20 breaths/ min, blood pressure was $150 / 90 \mathrm{~mm} \mathrm{Hg}$, and his weight was 217 pounds. He was not in severe distress. Tympanic membranes were clear with positive light reflexes. His throat was erythematous and without other lesions. His neck was supple with no meningeal signs. His lungs were clear to auscultation bilaterally. His abdomen was neither tender nor distended. There was no edema, cyanosis, or clubbing of his extremities. Computerized tomography (CT) of his sinuses showed mucosal thicken-

Submitted, revised, 10 March 2003.

From the Departments of Family Medicine (KS, EL), Nephrology (MA), and Pathology (JG), Louisiana State University Health Science Center, E. A. Conway Medical Center, Monroe, and Department of Pathology, Louisiana State University Health Science Center, Shreveport (MF). Address correspondence to Khaled Shafiei, MD, Department of Family Medicine, E. A. Conway Medical Center, 4864 Jackson St, Monroe, LA 71210 (e-mail: kshafiei@ hotmail.com).

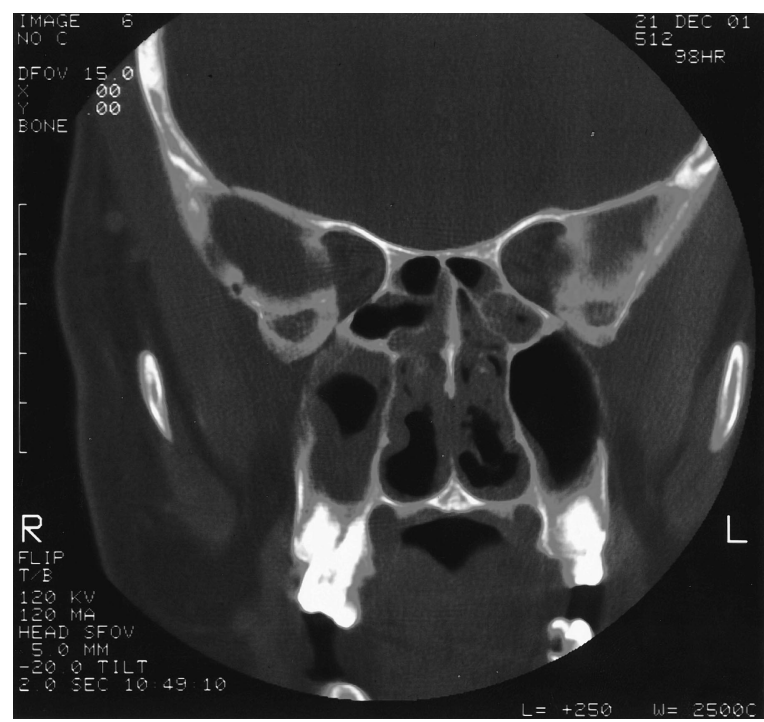

Figure 1. Focus of granulomatous inflammation, lung (hematoxylin and eosin; original magnification, $400 \times)$.

ing in all sinuses, including sphenoid, frontal, ethmoidal, and maxillary (Fig. 1). Laboratory studies included: WBC, $12.210^{3} / \mu \mathrm{L}$; hemoglobin, 12.3 $\mathrm{g} / \mathrm{dL}$; hematocrit, 0.345 ; platelets, $27110^{3} / \mu \mathrm{L}$; serum urea nitrogen, $21 \mathrm{mg} / \mathrm{dL}$; creatinine, $0.9 \mathrm{mg} /$ $\mathrm{dL}$; aspartate aminotransferase, $39 \mathrm{U} / \mathrm{L}$; alanine aminotransferase, $129 \mathrm{U} / \mathrm{L}$; alkaline phosphatase, $91 \mathrm{U} / \mathrm{L}$.

The admission diagnosis was pansinusitis that had failed outpatient treatment and a lung mass. The patient was started on $500 \mathrm{mg}$ /day levofloxacin administered intravenously. Magnetic resonance imaging of brain ruled out possible cavernous sinus thrombosis. CT of the chest showed a large, dominant, left upper lobe lesion abutting the pleura with two smaller noncalcified lesions in the left and right upper lobes; this was interpreted as suspicious for carcinoma of the lung (Figs. 2 and 3). CT of his abdomen and pelvis were negative. Tumor markers $(\alpha$-fetoprotein, prostate-specific antigen) were also negative. 


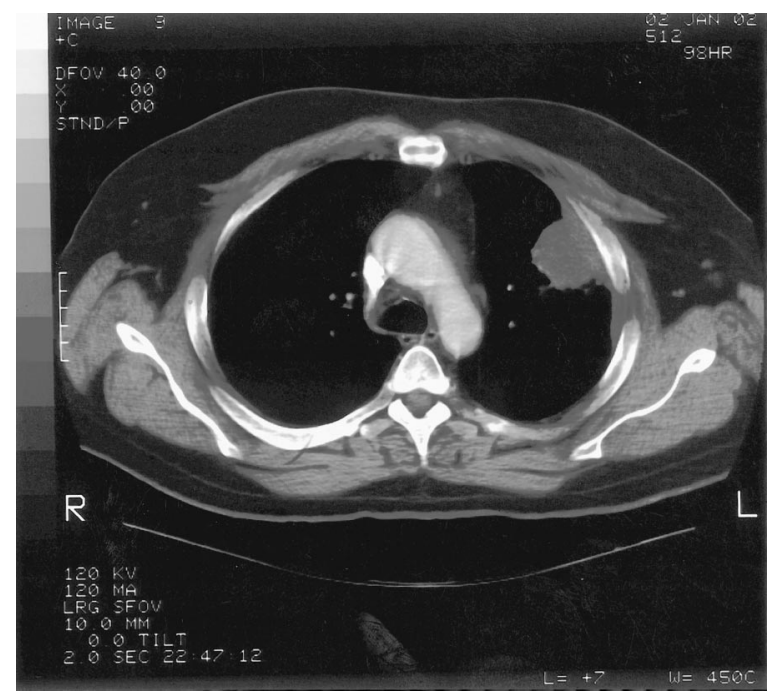

Figure 2. Coronal CT of sinuses demonstrating bilateral ethmoid and maxillary sinus disease.

On day 3, the patient complained of joint pain, mainly in the right shoulder and both hands. The patient's erythrocyte sedimentation rate and Creactive protein level were elevated. His rheumatoid factor level was $566 \mathrm{IU} / \mathrm{mL}$, and tests for antinuclear antibodies were negative. $\mathrm{He}$ was started on indomethacin and his symptoms improved in 2 days. CT-guided biopsy of the lung showed acute and chronic interstitial inflammation suggestive of a granulomatous process (Fig. 4). The patient was put in respiratory isolation until the results of 3 sets of cultures for mycobacteria came

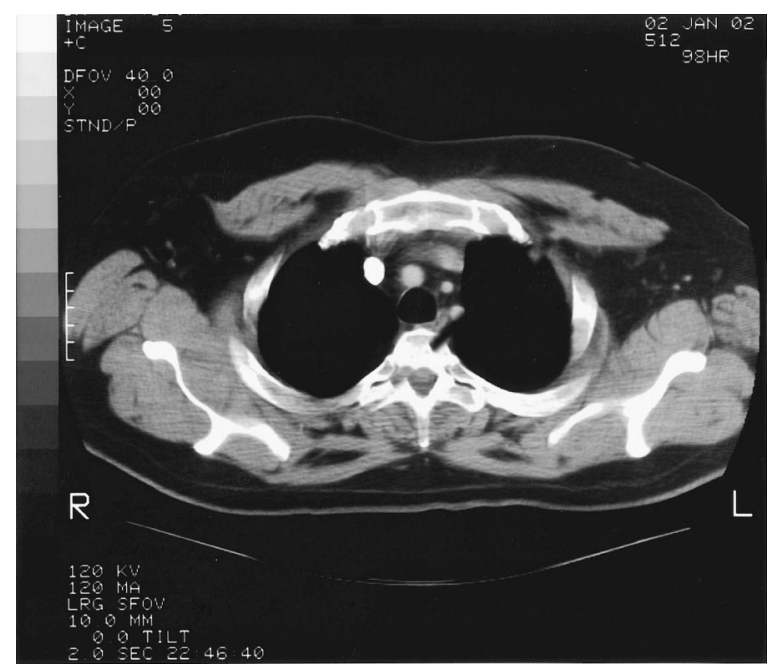

Figure 3. Chest CT demonstrating soft tissue mass in left upper lobe abutting the pleura. back negative. Sputum cultures were negative for fungus.

On day 10, the patient developed a painful ulcer on the left side of his tongue. Cultures were obtained from the lesion, and serum cytoplasmic antineutrophil cytoplasmic autoantibody (ANCA) levels were evaluated for WG. Results of the test for human immunodeficiency virus were negative. The patient's symptoms improved significantly with indomethacin and levofloxacin. He was discharged from the hospital on day 13 taking levofloxacin, ibuprofen, and loratadine with a follow-up appointment in infectious disease clinic for culture result and evaluation of his lung mass. The patient was also scheduled for an appointment with the ear-nose-throat clinic. C-ANCA level was still pending on discharge.

Two days after discharge, the patient came back with difficulty in swallowing, flank pain, and purpuric rash on both of his lower extremities up to the abdomen. He was also complaining of dysuria and blood in the urine. His urinalysis showed positive nitrites, large amounts of blood, and more than 100 red blood cells per high-power field; 11 to 25 WBCs per high-power field, and moderate bacteria. Repeat erythrocyte sedimentation rate was 55 $\mathrm{mm} / \mathrm{hr}$, C-reactive protein level was $22.4 \mathrm{mg} / \mathrm{dL}$, and rheumatoid factor was $1172.7 \mathrm{IU} / \mathrm{mL}$. He had another painful ulcer on his uvula. The patient was admitted again with the diagnosis of Wegener granulomatosis, although ANCA levels were still pending from previous admission. He was started on $125 \mathrm{mg}$ of intravenous methylprednisolone every 8 hours. A nephrologist was consulted.

\section{Discussion}

WG was first described by Klinger in 1933, followed by other investigators, including Rossle in 1933, Wegener in 1936 and 1939, and Ringertz in $1947 .^{1}$

WG is currently characterized as one of the ANCA-associated small vessel vasculitides. It is distinguished clinically by its predilection for affecting the upper and lower respiratory tracts and kidneys and by the histologic presence of necrosis, granulomatous inflammation, and vasculitis.

There is a strong and specific association with autoantibodies directed against proteinase 3 , a constituent of neutrophil azurophilic granules. ${ }^{2}$ The presence of such antibodies is a strong indicator for 


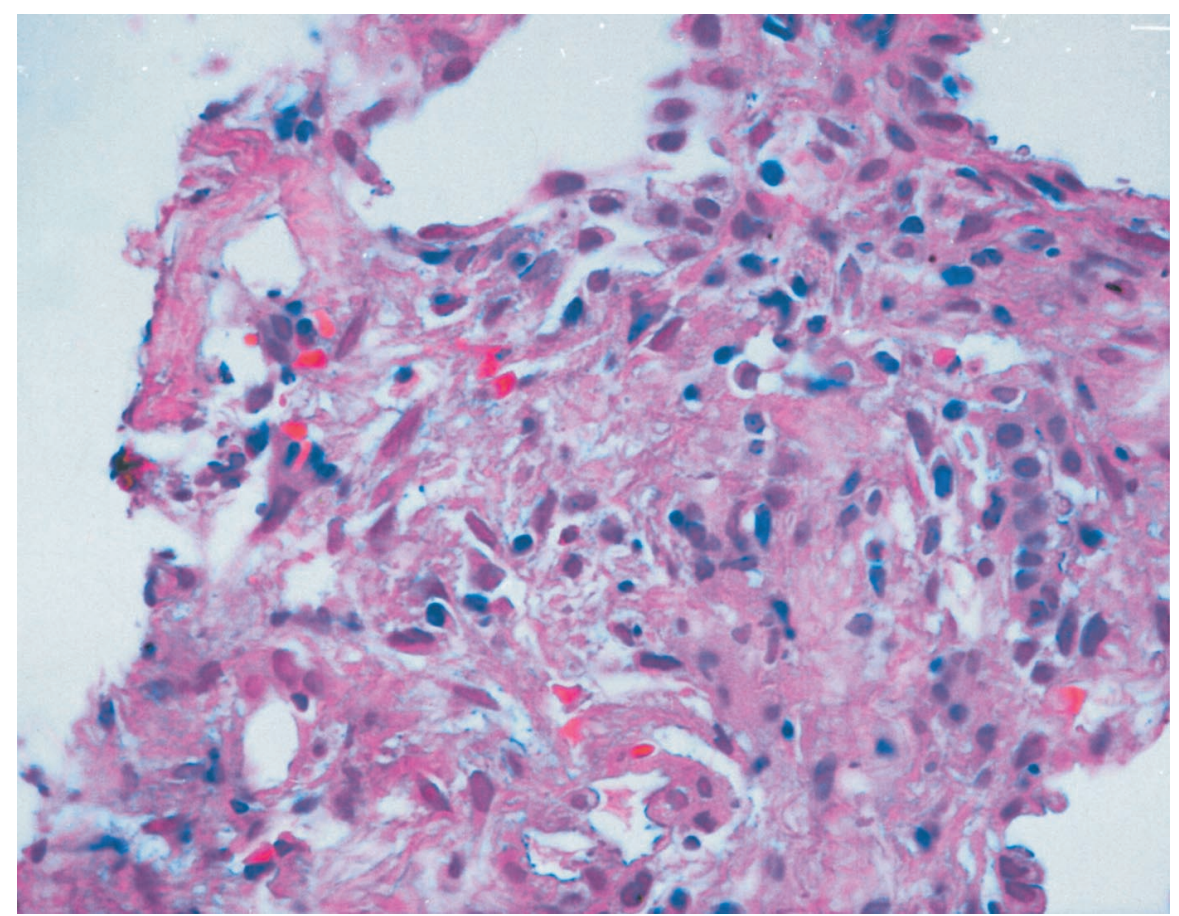

Figure 4. Chest CT demonstrating small noncalcified lesions in the left upper lobe.

a diagnosis of WG, but should not be used in place of a tissue diagnosis. The presence of ANCA is not required to make a diagnosis of WG by either the American College of Rheumatology (ACR) or the Chapel Hill Consensus Conference (CHCC) definitions. ${ }^{3}$ Occasionally, patients with infections, neoplasms, inflammatory bowel disease, sclerosing cholangitis and other rheumatologic diseases develop ANCA, but these are predominantly perinuclear ANCAs or exhibit an atypical staining pattern. ${ }^{4}$

Most patients are white, the sex distribution is equal, and most present in the fifth decade, although the age range extends to both extremes. ${ }^{5}$ The clinical presentation of WG can be so diverse that the list of differential diagnoses is vast, ranging from infections (fungal, bacterial, and mycobacterial) to other vasculitides, including HenochSchönlein purpura, sarcoidosis, Behcet syndrome, and malignancies. ${ }^{6}$

Unexplained constitutional symptoms are often part of the initial presentation. Fever and weight loss may be reported at the onset of the disease and more frequently during the course of the illness. ${ }^{5}$ The upper airway disease is the most common presenting feature of WG. This includes sinusitis, oral lesions (ulcer, gingivitis), otitis media, hearing loss, epistaxis, and saddle nose deformity; sinusitis is the most frequent initial presentation in about half to two thirds of patients with WG. ${ }^{5,7}$

Pulmonary involvement is one of the cardinal features of WG. It occurs in $45 \%$ of patients at presentation and $87 \%$ during the course of the disease. ${ }^{5}$ Cough, hemoptysis, and pleuritis are the most common pulmonary symptoms. The most common radiographic findings include pulmonary infiltrate $(67 \%)$ and nodules $(58 \%){ }^{5}$

Renal disease also may be seen as the initial presentation or during the course of the disease. Once present, renal disease may progress from asymptomatic and mild to fulminant glomerulonephritis within days or weeks, resulting in end-stage renal failure. ${ }^{7,8}$ Even with appropriate therapy, it may lead to chronic renal insufficiency and renal failure. $^{5}$

Ocular manifestations have been reported to occur in 28 to $58 \%$ of patients with WG, and they may be part of the initial presentation in 8 to $16 \%$ of patients. ${ }^{9,10}$ A complete ophthalmologic examination is an important part of the diagnostic evaluation. Any compartment of the eye may be affected. Keratitis, conjunctivitis, scleritis, episcleritis, nasolacrimal duct obstruction, uveitis, retroorbital pseudotumor with proptosis, retinal vessel occlusion, and optic neuritis have all been de- 
scribed..$^{5,9-11}$ Visual loss has been reported in as many as $8 \%$ of patients. ${ }^{5}$ CT or magnetic resonance imaging of the orbit and sinuses may provide useful anatomic information.

Other unusual presentations of WG include salivary gland, cutaneous, gastrointestinal, and cardiac involvement. ${ }^{5,7,8,11-18}$

\section{Treatment}

Traditionally, initial therapy of WG consists of daily oral cyclophosphamide-corticosteroid combination therapy. This treatment has been quite effective in inducing remission in more than $90 \%$ of patients who adhere to this regimen; approximately $75 \%$ experience complete remission. ${ }^{5,19,20}$ The mean time to complete remission is 12 months, with occasional patients requiring treatment for more than 2 years before all symptoms have resolved. Therefore, patients should not be considered nonresponders until they have been monitored on this regimen for more than several months. Response to this regimen is defined as a lessening or resolution of the inflammatory manifestations. ${ }^{19}$ This is important because new patients may have persistent abnormalities that are not caused by active disease. For example, patients in whom the systemic symptoms and signs resolve and their urinalyses become inactive are considered to be in remission, but they may have persistent or even slowly worsening renal insufficiency.

The use of aggressive immunotherapy in this disease is justified because survival in patients with untreated WG is extremely poor; up to $90 \%$ of patients die within 2 years, usually because of respiratory or renal failure. ${ }^{5,19,20}$ Mortality, however, can be significantly reduced with the introduction of a cyclophosphamide-corticosteroid therapy combination. The two routes of administration are oral and intravenous. Direct comparisons between oral and intravenous cyclophosphamide also have been performed in small prospective and randomized trials. ${ }^{20-23}$ Intravenous and oral cyclophosphamide have similar efficacy in terms of inducing remission. ${ }^{22,23}$ Intravenous therapy was associated with less toxicity but a trend toward a higher rate of relapse. ${ }^{23,24}$ In the medical literature, the daily oral cyclophosphamide and corticosteroid regimen is the preferred method of managing these patients. However, in patients who have limited or non-lifethreatening disease, monthly intravenous cyclophosphamide pulse therapy can be effective and can minimize some of the side effects. ${ }^{21,22,24}$ Pulse therapy is given in conjunction with prednisone, usually starting at $1 \mathrm{mg} / \mathrm{kg} /$ day and given at this dosage for at least 1 month, then tapering by 5 to $10 \mathrm{mg}$ per week until prednisone is stopped or the patient is placed on maintenance therapy of 5 to 15 mg every other day.

Other therapies for WG include methotrexate and prednisone, which is another alternative for patients with active but not immediately lifethreatening disease and normal or near-normal renal function. ${ }^{25,26}$ This particular regimen may be attractive in patients who have limited bone marrow reserve from past cyclophosphamide therapy or a history of cyclophosphamide-induced bladder injury. Prednisone alone is not a recommended therapy for WG. ${ }^{5,24}$

In those with severe disease at presentation, pulmonary hemorrhage, or worsening disease despite immunosuppressive therapy plasmapheresis may be indicated. ${ }^{27,28}$ There is not much clinical data to address the long-term outcome in patients who undergo plasmapheresis for WG. ${ }^{29,30}$

In our case, the patient began therapy with monthly intravenous cyclophosphamide pulse therapy. The use of monthly intravenous pulse therapy is primarily to lower the overall cumulative dose of cyclophosphamide and thereby avoid some of the toxic side effects of the oral regimen. Our patient responded to intravenous pulse cyclophosphamide therapy and steroids with marked improvement in his urinalysis, decreased numbers of red blood cells per high power field, improvement in his serum creatinine level, and improvement of his inflammatory symptoms. He received a total of 6 months of intravenous pulse therapy, which he tolerated quite well, and has so far not had any signs of relapse.

\section{References}

1. Lie JT. Illustrated histologic classification criteria for selected vasculitis syndromes. Arthritis Rheum 1990; 33:1074-87.

2. Hewins P, Tervaert JW, Savage CO, Kallenberg CG. Is Wegener's granulomatosis an autoimmune disease? Curr Opin Rheumatol 2000;12:3-10.

3. Watts RA. Wegener's granulomatosis: unusual presentations. Hosp Med 2000;61:250-3.

4. Gross WL. Antineutrophil cytoplasmic autoantibody testing in vasculitides. Rheum Dis Clin North Am 1995;21:987-1011.

5. Hoffman GS, Kerr GS, Leavitt RY, et al. Wegener 
granulomatosis: an analysis of 158 patients. Ann Intern Med 1992;116:488-98.

6. Schmacher HR, Klippel JH, Koopman WJ, editors. Primer on the rheumatic disease. Atlanta: Arthritis Foundation; 1993. p. 136-48.

7. Fauci AS, Haynes BF, Katz P, Wolff SM. Wegener's granulomatosis: prospective clinical and therapeutic experience with 85 patients for 21 years. Ann Intern Med 1983;98:76-85.

8. Fauci AS, Wolff SM. Wegener's granulomatosis: studies in eighteen patients and a review of the literature. Medicine (Baltimore) 1973;52:535-61.

9. Anderson G, Coles ET, Crane M, et al. Wegener's granuloma. A series of 265 British cases seen between 1975 and 1985. A report by a sub-committee of the British Thoracic Scociety Research Committee. Q J Med 1992;83:427-38.

10. Bullen CL, Liesegang TJ, McDonald TJ, DeRemee RA. Ocular complications of Wegener's granulomatosis. Ophthalmology 1983;90:279-90.

11. Haynes BF, Fishman ML, Fauci AS, Wolff SM. The ocular manifestations of Wegener's granulomatosis. Fifteen years experience and review of literature. Am J Med 1977;63:131-41.

12. Klinger H. Grenzforman der periarteritis nodosa. Frankfurt Z Pathol 1931;42:455-80.

13. De Oreo GA. Wegener's granulomatosis. Arch Dermatol 1960;81:169-74.

14. Walton EW. Giant-cell granuloma of the respiratory tract (Wegener's Granulomatosis). Br Med J 1958;2:265-70.

15. Aymard B, Bigard MA, Thompson H, Schmutz JL, Finet JF, Borrelly J. Perianal ulcer: an unusual presentation of Wegener's granulomatosis. Report of a case. Dis Colon Rectum 1990;33:427-30.

16. Wilson RT, Dean PJ, Upshaw JD, Wruble LD. Endoscopic appearance of Wegener's granulomatosis involving the colon. Gastrointest Endosc 1987; 33:388-9.

17. Grant SC, Levy RD, Venning MC, Ward C, Brooks NH. Wegener's granulomatosis and the heart. $\mathrm{Br}$ Heart J 1994;71:82-6.

18. Schiavone WA, Ahmad M, Ockner SA. Unusual cardiac complications of Wegener's granulomatosis. Chest 1985;88:745-8.

19. Hoffman GS, Leavitt RY, Fleisher TA, Minor JR, Fauci AS. Treatment of Wegener's granulomatosis with intermittent high-dose intravenous cyclophosphamide. Am J Med 1990;89:403-10.

20. Gross WL, Rasmussen N. Treatment of Wegener's granulomatosis: the view from two non-nephrologists. Nephrol Dial Transplant 1994;9:1219-22.

21. Guillevin L, Cordier J-F, Lhote F, et al. A prospective multicenter randomized trial comparing steroids and pulse cyclophosphamide versus steroids and oral cyclophosphamide in the treatment of generalized Wegener's granulomatosis. Arthritis Rheum 1997; 40:2187-98.

22. Haubitz M, Schellong S, Gobel U, et al. Intravenous pulse administration of cyclophosphamide versus daily oral treatment in patients with antineutrophil cytoplasmic antibody-associated vasculitis and renal involvement: a prospective randomized study. Arthritis Rheum 1998;41:1835-44.

23. Adu D, Pall A, Luqmani RA, et al. Controlled trial of pulse versus continuous prednisone and cyclophosphamide in the treatment of systemic vasculitis. QJM 1997;90:401-9.

24. de Groot K, Adu D, Savage CO. The value of pulse cyclophosphamide in ANCA-associated vasculitis: meta-analysis and critical review. Nephrol Dial Transplant 2001;16:2018-27.

25. Langford CA, Sneller MC, Hoffman GS. Methotrexate use in systemic vasculitis. Rheum Dis Clin North Am 1997;23:841-53.

26. Langford CA, Talar-Williams C, Sneller MC. Use of methotrexate and glucocorticoids in the treatment of Wegener's granulomatosis. Long-term renal outcome in patients with glomerolonephritis. Arthritis Rheum 2000;43:1836-40.

27. Pusey CD, Rees AJ, Evans DJ, Peters DK, Lockwood CM. Plasma exchange in focal necrotizing glomerulonephritis without anti-GBM antibodies. Kidney Int 1991;40:757-63.

28. Cole E, Cattran D, Magil A, et al. A prospective randomized trial of plasma exchange as additive therapy in idiopathic crescentic glomerulonephritis. Am J Kidney Dis 1992;20:261-9.

29. Zauner I, Bach D, Braun N, et al. Predictive value of initial histology and effects of plasmaphoresis on long-term prognosis of rapidly progressive glomerulonephritis. Am J Kidney Dis 2002;39:28-35.

30. Guillevin L, Lhote F, Cohen P, et al. Corticosteroids plus pulse cyclophosphamide and plasma exchanges versus corticosteroids plus pulse cyclophosphamide alone in the treatment of polyarteritis nodosa and Churg-Strauss syndrome patients with factors predicting poor prognosis. A prospective, randomized trial in sixty-two patients. Arthritis Rheum 1995;38: $1638-45$. 